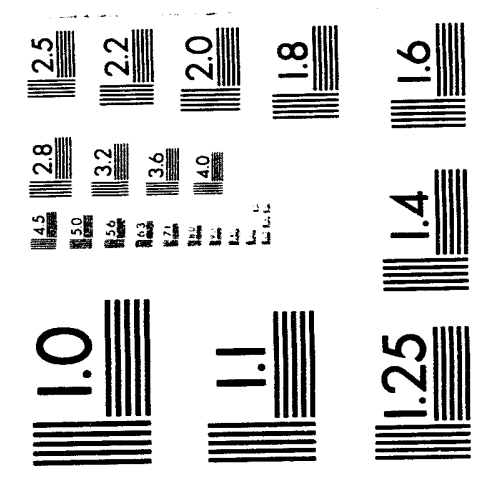



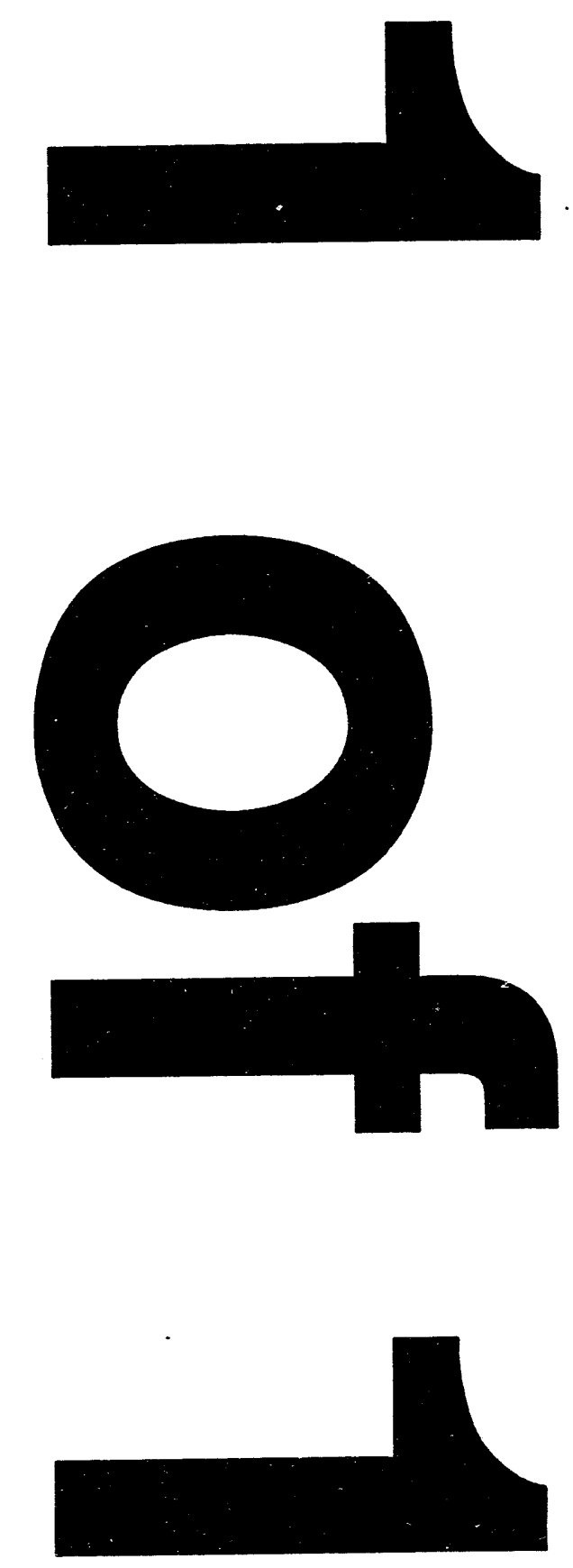


\section{INSTITUTE FOR \\ FUSION STUDIES}

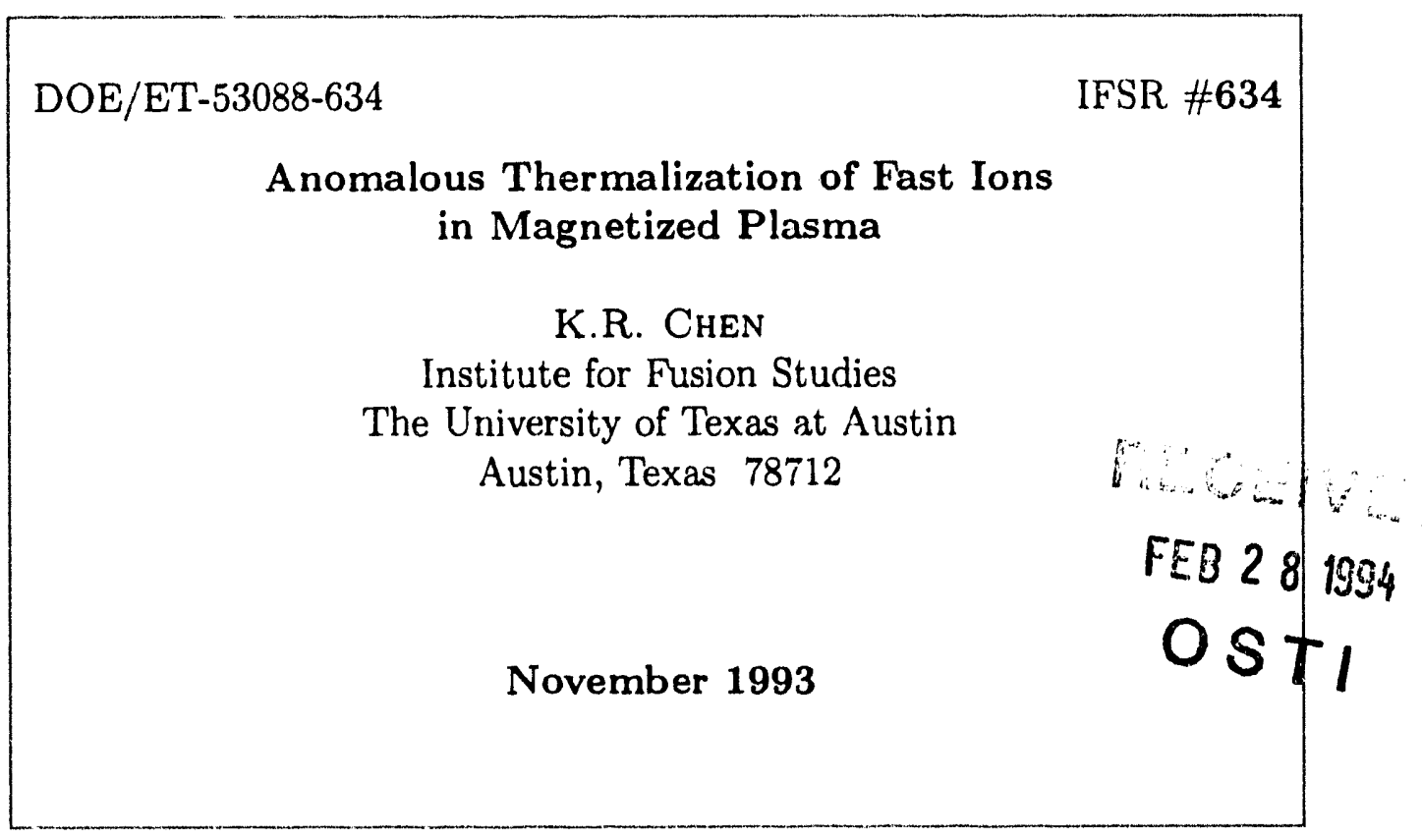

\section{THE UNIVERSITY OF TEXAS}

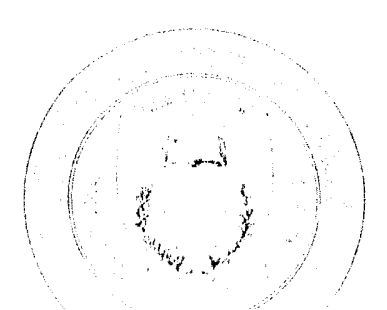

\section{AUSTIN}




\title{
Anomalous Thermalization of Fast Ions in Magnetized Plasma
}

\author{
K.R. Chen \\ Institute for Fusion Studies \\ The University of Texas at Austin \\ Austin, Texas 78712
}

\begin{abstract}
A novel anomalous process causing the perpendicular energy of fast ions to be thermalized and lost on average to bulk ion heating, instead of classical slowing down and bulk electron heating, is investigated with PIC simulations. More than half of the fast ions are slowed down to the thermal ion level, although some are heated to twice their birth energy. The fast ion density perturbation is large. This process is excited by a new two-gyro-stream instability and may continually occur in a burning plasma. The implications for fusion ignition and fast ion confinement are assessed.
\end{abstract}

\section{DISCLAIMER}

This report was prepared as an account of work sponsored by an agency of the United States Government. Neither the United States Government nor any agency thereof, nor any of their employees, makes any warranty, express or implied, or assumes any legal liability or responsibility for the accuracy, completeness, or usefulness of any information, apparatus, product, or process disclosed, or represents that its use would not infringe privately owned rights. Reference herein to any specific commercial product, process, or service by trade name, trademark, manufacturer, or otherwise does not necessarily constitute or imply its endorsement, recommendation, or favoring by the United States Government or any agency thereof. The vicws and opinions of authors expressed herein do not necessarily state or reflect those of the United States Government or any agency thereof. 
Fast ions exist in laboratory, fusion [1], space, and astrophysical [2] plasmas. In a fusion plasma, the energy needed to sustain ignition is provided by the isotropic shell-distributed fast ions produced from thermonuclear fusion reactions. Thus, the anomalous behavior of fast ions due to collective instabilities, as well as the implications for their confinement, are critically important issues for the performance of a burning fusion reactor.

In the past; research on fast-ion-driven instabilities has focused on mechanisms based on an imbalanced population (e.g., inverse Landau damping). The time scales of the waves (e.g., the Toroidal Alfvén Eigenmode $[3,4]$ ) are usually longer than the inverse ion cyclotron frequency. Furthermore, the fast ions are assumed to be distributed smoothly in real space and to have a slowing-down distribution in energy, as predicted classically, where the collision time is much longer than the inverse ion cyclotron frequency.

Recently, intense localized harmonic ion cyclotron emission induced by fast ions has become an active area of research, with both theoretical studies [5]-[9] and experimental observations [10]-[12]. In particular, the mechanism of the two-gyro-stream instability (a)so called the two-energy-stream cyclotron instability [5]), which is driven by the fast ions due to a weak relativistic mass effect, has been shown [6] by means of both simulation and theory for example, through a comparison of the relative spectral amplitudes of the low harmonics - to be consistent with the experimental observations.

In this Letter, I investigate a novel anomalous process that causes the perpendicular energy of fast ions to be thermalized and lost on average to bulk ion heating, instead of classical slowing down [13] and bulk electron heating $[1,13]$. My simulations show that the fast ion dynamics are dramatically affected in both real and velocity space, in contrast to other recently proposed mechanisms [7]-[9], which predict effects that are weaker and occur in narrower regimes of phase space. This process is excited by the two-gyro-stream instability, and persists even when the external confining magnetic field is nonuniform. The 
investigation was carried out by means of Particle-In-Cell simulations with a quiet start $[14,15]$. The simulations imply that the anomalous process may continually occur in a burning plasma. When the weak relativistic mass effect is turned off, the observed anomalous phenomena disappear. The anomalous process has implications for burning conditions and their confinement.

The two-gyro-stream instability arises from the coupling of a fast ion Bernstein branch and its corresponding slow ion Bernstein branch. Due to the relativistic mass dependence, the harmonic cyclotron frequency of the fast ions is slightly smaller than the harmonic cyclotron frequency of the slow ions. It gives rise to a "two-streaming" process in gyrospace (i.e., gyrophase vs. perpendicular momentum). The peak growth rate $\omega_{i}[5]$ of the instability for the system considered here is $\omega_{i} / \ell_{o} \omega_{c \Omega}=(\sqrt{3} / 2)(\gamma-1)^{1 / 3}\left(\omega_{p f}^{2} / \omega_{p \ell}^{2}\right)^{1 / 3}\left(\left\langle J_{\ell_{f}}\left(k \rho_{f}\right)\right\rangle\right)^{1 / 3}\left(\ell_{f}^{2} \omega_{c f}^{2}-\right.$ $\left.\omega_{c s}^{2}\right)^{1 / 3} /(k c)^{2 / 3}$, with the real frequency of the wave given by $\omega_{r} / \ell_{f} \omega_{c f}=1+(1 / \sqrt{3})\left(\omega_{i} / \ell_{g} \omega_{c s}\right)$; here, $\ell$ is the harmonic number, $\omega_{c}$ is the relativistic cyclotron frequency, $\gamma$ is the fast ion Lorentz factor, $\omega_{p}$ is the plasma frequency, $k$ is the wavenumber, $c$ is the speed of light, $J_{l_{f}}$ is the Bessel function of the first kind of order $\ell_{f},\langle\rangle$ represents the integration over a shell velocity distribution, $\rho$ is the Larmor radius, and the subscripts $f$ and $s$ indicate fast and slow ions, respectively. The fast ions give up energy to the wave, and the wave to the slow ions, which brings their frequencies closer. The wave growth ceases when the difference of $\omega_{r}$ and $\ell_{f} \omega_{c f}$ vanishes due to the change in $\gamma$. Hencr, the rate of fast ion energy loss is estimated [5] to be $\eta=3^{-1 / 2}\left(\omega_{i} / \ell_{f} \omega_{c f}\right)(\gamma-1)^{-1}$. In the simulations, the coupling of the first proton cyclotron harmonic and the second bulk deuteron cyclotron harmonic is dominant.

A one-space-and-three-momentum-dimensional simulation code is employed. The saturated wave potential is expected to be smaller than the slow ion temperature. Because the wavelength is much larger than the plasma Debye length, the saturated field energy should be very small. Thus, I adopt the quiet start technique for the simulation to have low noise. Also, the model is simplified in order to make the simulation affordable even while the essen- 
tial physics is retained. Because the modes with vanishing parallel wave number $\left(k_{z}=0\right)$ are more unstable than the $k_{z} \neq 0$ modes [5], the wave vectors are across the external magnetic field, which is taken along the $\hat{z}$ direction. Fortunately, $k_{z}=0$ is usually a good assumption $[16,17]$ for cyclotron waves in toroidal geometry as well. Inclusion of an electromagnetic Alfvén mode may not be important. Its excitation requires the extra condition $k v_{A}=\ell \omega_{c}$, where $v_{A}$ is the Alfvén velocity. Also, almost all the energy of the Alfvén mode is in its magnetic component whereas an electric field is needed to change particle energy. Therefore, only the effects caused by electrostatic ion Bernstein modes are considered. The spatially localized feature as inferred from the experimental observations makes a periodical boundary condition suitable. The electrons are treated with a dielectric, but the kinetic ions can be treated relativistically or classically, which offers an important check on the importance of the weak relativistic mass effect.

The physical parameters for the simulation are as follows: $\omega_{p D} / \omega_{c D}=19.2, n_{p} / n_{D}=5 \times$ $10^{-3}, E_{p} / T_{D}=147$, and $\gamma=1.0157$ (which corresponds to $14.7 \mathrm{MeV}$ protons produced from deuterium-helium-3 reactions) with $4 \%$ energy spread (full-width at half-maximum). Here $n$ is the density, $E$ is the energy, $T$ is the temperature, and the subscripts $p$ and $D$ represent the shell-distributed protons and Maxwellian deuterons, respectively. The instability is absolute [18] because the peak growth rate for the first proton harmonic, $8.2 \times 10^{-3} \omega_{c D}$, is larger than the critical rate for absolute instability, $3.8 \times 10^{-3} \omega_{c D}$. The system length is 4096 grids, with $d x$ the grid size (normalized to unity). The fast ion gyroradius is $\rho_{p}=222 d x$. Time is normalized to $\omega_{c D}^{-1}$, and one time step is 0.02 . The proton mass is normalized to have unit value. The unit charge is the proton charge. Energy is normalized to the total system energy. The simulations use 177,146 superparticles for the deuterons and 21,600 for the protons. Modes with mode numbers from 1 to 15 are kept; the wave number $k$ is equal to $2 \pi / 4096$ times the mode number. The initial system noise is about $1 \times 10^{-8}$.

The simulations show that the field energy of the wave grows exponentially from $1 \times 10^{-10}$, 
with a growth rate $\omega_{i}=0.8 \%$ that agrees with the theoretical prediction. It reaches a saturation level of $8 \times 10^{-6}$ at $t=1000$, as shown in Fig. 1. The wave potential divided by the slow ion temperature is $0.09,0.28$, and 0.13 at saturation, respectively, for modes 6,7 , and 8 (which are the modes of largest field energy). From the power spectrum, these modes have peaks at the real frequencies $1.980,1.974$, and 1.967 , respectively. Due to the collective instability, the fast ions have lost kinetic energy, on average, of about $20 \%$ at the saturated peak and $12 \%$ at the end of the simulation at $t=3200$, which losses are consistent with the theoretical estimate of $15 \%$. Figure 1 also shows that almost all the energy loss goes into bulk ion heating. The resultant slow ion distribution becomes non-Maxwellian and has an energetic tail. When I turn off the weak relativistic mass effect by using Newton's equation instead of the Lorentz equation for the particle motion, there are no wave growth, no fast ion energy loss, and no energy gained by the slow ions, as shown on Fig. 1. This verifies that the anomalous process is caused by the weak relativistic mass effect.

While the fast ions lose energy on average, due to the collective cyclotron instability, some of them gain energy from the waves and some of them lose energy, depending on their gyrophases. The fast ion energy distribution at various times is shown in Fig. 2. The energy spread quickly increases and reaches about $100 \%$ at the time of saturation. After that, the energy spread remains about the same, while some of the overshot fast ions gain energy back and others lose (or gain) more energy. At the end of the simulation, more than half of the fast ions are slowed down up to the thermal ion level, although some of them are heated up to twice their birth energy. A similar phenomenon is observed during neutralbeam-injection experiments in tokamaks $[19,20]$. The two-gyro-stream instability occurs in the perpendicular direction and has no effect on the parallel momentum. Figure 3 shows that, due to the collective instability, the perpendicular momentum distribution function of the fast ions changes from a shell distribution toward a Maxwellian distribution. It is also observed that the particles bunch in their gyrophase. An electrostatic wave results from the 
real space perturbation of the charged particles and vice versa. Figure 4 shows that the fast ion density, which was initially uniform, develops a sizeable perturbation. The density perturbation reaches $100 \%$ at wave saturation and remains at the $50 \%$ level at the end of the simulation.

This anomalous process is strong. As it creates a large spread in the fast ion energy and thermalizes their perpendicular momentum, it removes fast ion kinetic energy and, at the same time, heats the bulk ions. Bulk ion heating favors the occurrence of the hot ion mode [21] and sustained ignition in a fusion reactor. The thermalization dramatically changes the pitch angle and banana width of the fast ions and thus may affect their confinement. On the other hand, it also produces a large perturbation in the fast ion density across the confining magnetic field. In a realistic device, the unperturbed density is usually higher at the plasma center. Thus, a ponderomotive force may be produced that can induce anomalous transport across the confining magnetic field. Although this may provide a means for ash removal and for the weakening of instabilities driven by a real-space gradient, it may also cause serious problems for a burning plasma, such as loss of the fast ions for sustaining ignition.

The basic mechanism for the cubic two-gyro-stream instability is due to the difference of the fast ion cyclotron harmonic frequency and its corresponding slow ion cyclotron harmonic frequency. A nonuniform magnetic field does not change this relationship, although it may localize the waves. Two simulations were also carried out with a perturbed magnetic field that had a $1.6 \%$ and $5 \%$ peak-to-peak sinusoidal nonuniformity, respectively, with other parameters the same. The ratio of the fast ion gyroradius to the nonuniformity scale length in the $5 \%$ case is comparable to that in typical tokamaks.

Figure 5 shows the fast ion energy distribution at the end of simulations for cases with different amplitudes of the magnetic field nonuniformity. The large energy spreads attained are essentially the same in all the cases. At the end of the simulations, the fast ions lose $9 \%$ and $7 \%$ of their energy in the $1.6 \%$ and $5 \%$ nonuniformity cases, respectively. Again, almost 
all of the lost energy goes into bulk ion heating. The simulations also show the thermalization of the fast ion perpendicular momentum, the large fast ion density perturbation, and other phenomena observed in the uniform simulations. More wave Fourier components are observed to become unstable in the nonuniform magnetic field, but their peak growth rates are smaller than those for the uniform magnetic field case. The power spectrum for the $5 \%$ nonuniformity case shows that each wave Fourier mode from 1 to 12 (note that the Fourier modes from 13 to 15 were not retained in order to reduce the numerical noise level) has peaks at the frequency 2.02 or 1.93 or both. Within the numerical resolution, the wave field perturbations are localized and absolutely unstable. This feature indicates the existence of cyclotron eigenmodes and is being further studied. As far as the anomalous process, the simulation results are insensitive to the magnetic field nonuniformity.

The initial distribution used for the fast ions is valid for a burning plasma at its initial stage or for the plasma in the edge region [6]-[12] where fast ions are pushed out by MHD activity. Further numerical experiments were performed in order to determine whether this process may continually occur in a burning plasma. After the waves have saturated and the fast ions are thermalized, I add new-born fast ions into the system. In the first case, I add in new-born fast ions with density of $5 \times 10^{-4} n_{D}$ right after $t=1600$. The simulation shows that all the previously observed anomalous phenomena occur for the new-born fast ions, within a shorter time scale of $200 w_{c D}^{-1}$. In the second case, I gradually add in new-born fast ions at times right after 1600,1700,1800,1900, and 2000, respectively, each time with the density of $1 \times 10^{-4} n_{D}$. Figure 6 shows the energy distribution of the new-born fast ions at different times. Again, all the previously observed anomalous phenomena - such as the large energy spread, the thermalized perpendicular momentum distribution, and the large density perturbation - occur for the new-born fast ions, in a shorter time. These simulation results imply that this process may continually occur in a burning plasma. Note that the rates of fusion reaction, ash removal, and collisions, as well as nonideal effects, should be 
taken into account in simulating a realistic device.

In summary, I have investigated the novel anomalous process that causes the perpendicular energy of fast ions to be thermalized and lost to bulk ion heating, which is highly favorable in a fusion device for the operation of the hot ion mode and for sustained ignition. There could be some additional favorable effects such as ash removal and the weakening of other instabilities, but also an unfavorable effect (the loss of some accelerated fast ions). More experiments that measure the fast ion energy distribution and correlate it with the cyclotron emission spectrum could confirm this anomalous process. Not only is this anomalous process important for fusion research, it may also serve in space and astrophysical plasmas as a means of thermalizing fast ions while accelerating some of them to even higher energy and as a mechanism for ion cyclotron emission.

The author appreciates comments from J.W. Van Dam on the manuscript. This work was supported by the U.S. Department of Energy contract \#DE-FG05-80ET-53088. 


\section{References}

[1] H.P. Furth et al., Nucl. Fusion 30, 1799 (1990), and references therein.

[2] D.B. Melrose, Instabilities in space and laboratory plasmas (Cambridge University Press, London, 1986), and references therein.

[3] F. Zonca and L. Chen, Phys. Rev. Lett. 68, 592 (1992).

[4] M.N. Rosenbluth, H.L. Berk, J.W. Van Dam, and D.M. Lindberg, Phys. Rev. Lett. 68, 596 (1992).

[5] K.R. Chen, Bull. Am. Phys. Soc. 37, 1421 (1992), and Phys. Lett. A 181, 308 (1993).

[6] K.R. Chen, W. Horton, and J.W. Van Dam, submitted to Phys. Fluids B.

[7] R.O. Dendy, C.N. Lashmore-Davies, and K.F. Kam, Phys. Fluids B 4, 3996 (1992); also 5, 1937 (1993).

[8] V. Arunasalam, G.J. Greene, and K.M. Young, Bull. Am. Phys. Soc. 37, 1605 (1992).

[9] B. Coppi, Phys. Lett. A 172, 439 (1993); also, private communication (April, 1993).

[10] G.A. Cottrell and R.O. Dendy, Phys. Rev. Lett. 60, 33 (1988), and references therein.

[11] P. Schild, G.A. Cottrell, and R.O. Dendy, Nuclear Fusion 29, 834 (1989).

[12] JET Team, Nuclear Fusion 32, 187 (1992); also, A. Gibson, private communication (April, 1992).

[13] Ya.I. Kolesnichenko, Nucl. Fusion 20, 727 (1980), and references therein.

[14] J.M. Dawson, Rev. Mod. Phys. 55, 403 (1983). 
[15] C.K. Birdsall and A.B. Langdon, Plasma Physics via Computer Simulation (Adam Hilger, New York, 1991), p. 394.

[16] A.B. Mikhailovskii, Theory of Plasma Instabilities (Consultants Bureau, New York, 1974), Vol. 2, p. 151.

[17] T.D. Kaladze and A.B. Mikhailovskii, Sov. J. Plasma Phys. 1, 128 (1975).

[18] T.H. Stix, Waves in Plasmas (American Institute of Physics, New York, 1992), p. 220.

[19] M. Seki, et al., Phys. Rev. Lett. 62, 1989 (1989).

[20] P. Beiersdorfer, R. Kaita, R.J. Goldston, Nucl. Fusion 24, 487 (1984).

[21] JT-60 team, Fourteenth Int'l Conf. on Plasma Physics and Controlled Nuclear Fusion Research (IAEA: Würzburg, Germany, 1992), Vol. 1, p. 57. 


\section{Figure Captions}

1. The history of the field energy and the kinetic energies of the fast ion and the slow ion for the relativistic and nonrelativistic runs, respectively.

2. The kinetic energy distribution of the fast ion at $t=0,800,1000$, and 3200 , respectively. The curve for the $t=0$ case has been multiplied by 0.15 .

3. The perpendicular momentum distribution of the fast ion at $t=0,800,1000$, and 3200 , respectively. The thermal momentum of the Maxwellian distribution, for comparison, is 300 . The curve for the $t=0$ case has been multiplied by 0.5 .

4. The fast ion density vs. position, $x$, at $t=1000$. The density is summed over every 4 grids. The average density is about 21 .

5. The kinetic energy distribution of the fast ion at $t=3200$ for the cases with uniform, $1.6 \%$ nonuniform, and $5.0 \%$ nonuniform magnetic fields, respectively.

6. The kinetic energy distribution of the new-born fast ions at birth, $t=1800,2000$, and 2200 , respectively. They are added in right after $t=1600,1700,1800,1900$, and 2000 , respectively, each time with a density of $1 \times 10^{-4} n_{D}$ and a shell distribution of $\gamma-1=$ 0.0157 . The curve for the birth cases has been multiplied by 0.3 . 


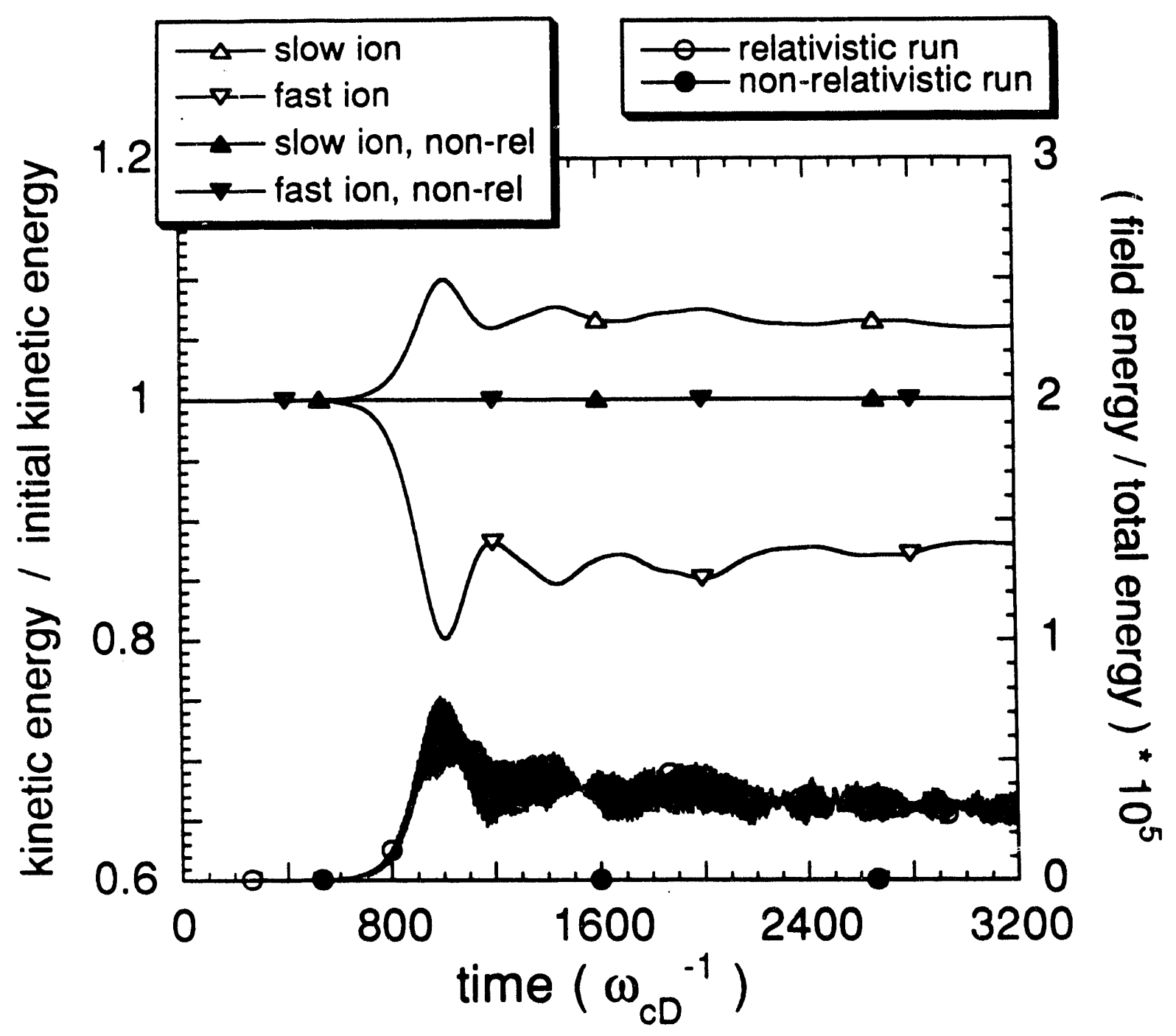

Fig. 1. by Chen 


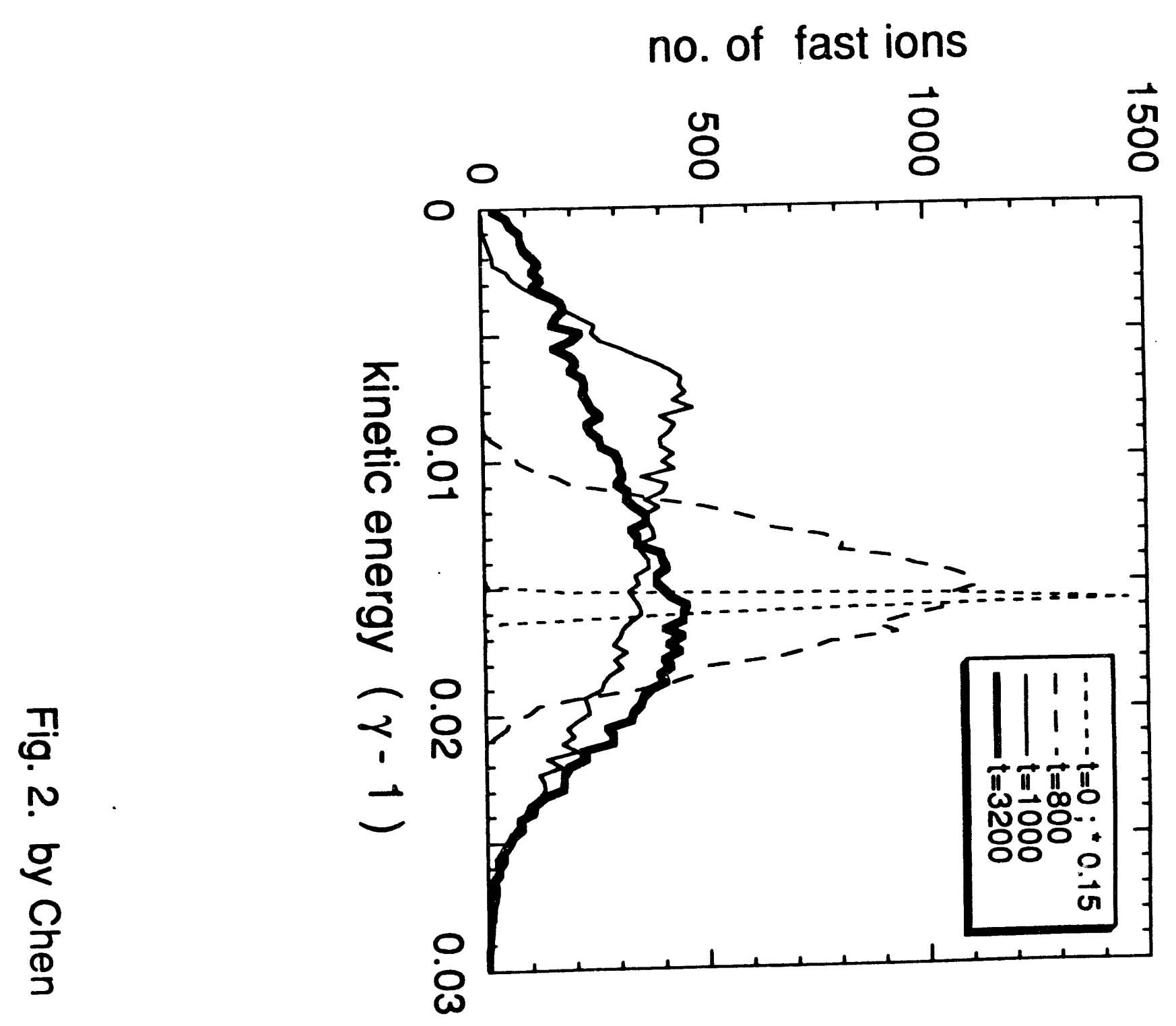


distribution function
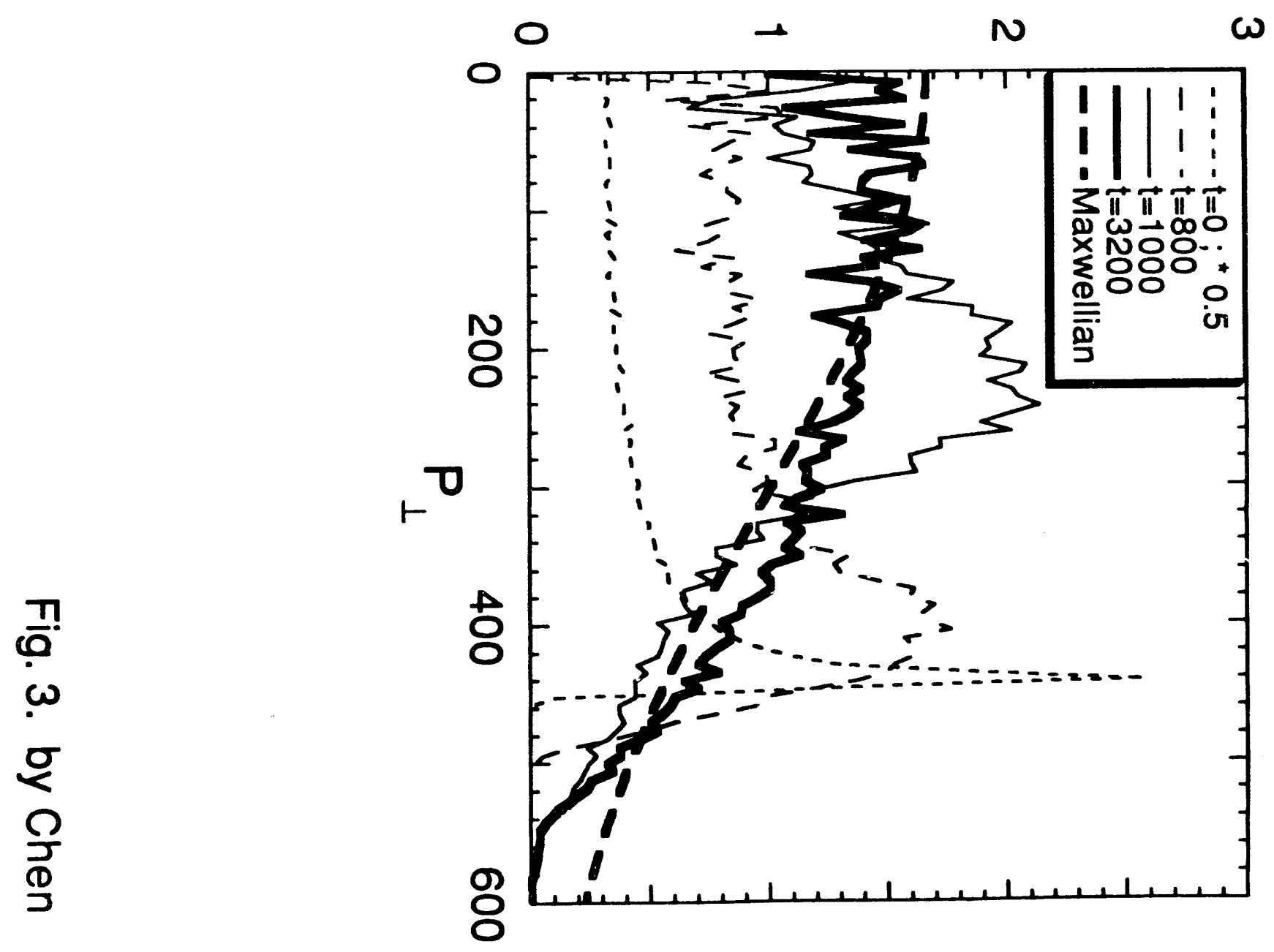


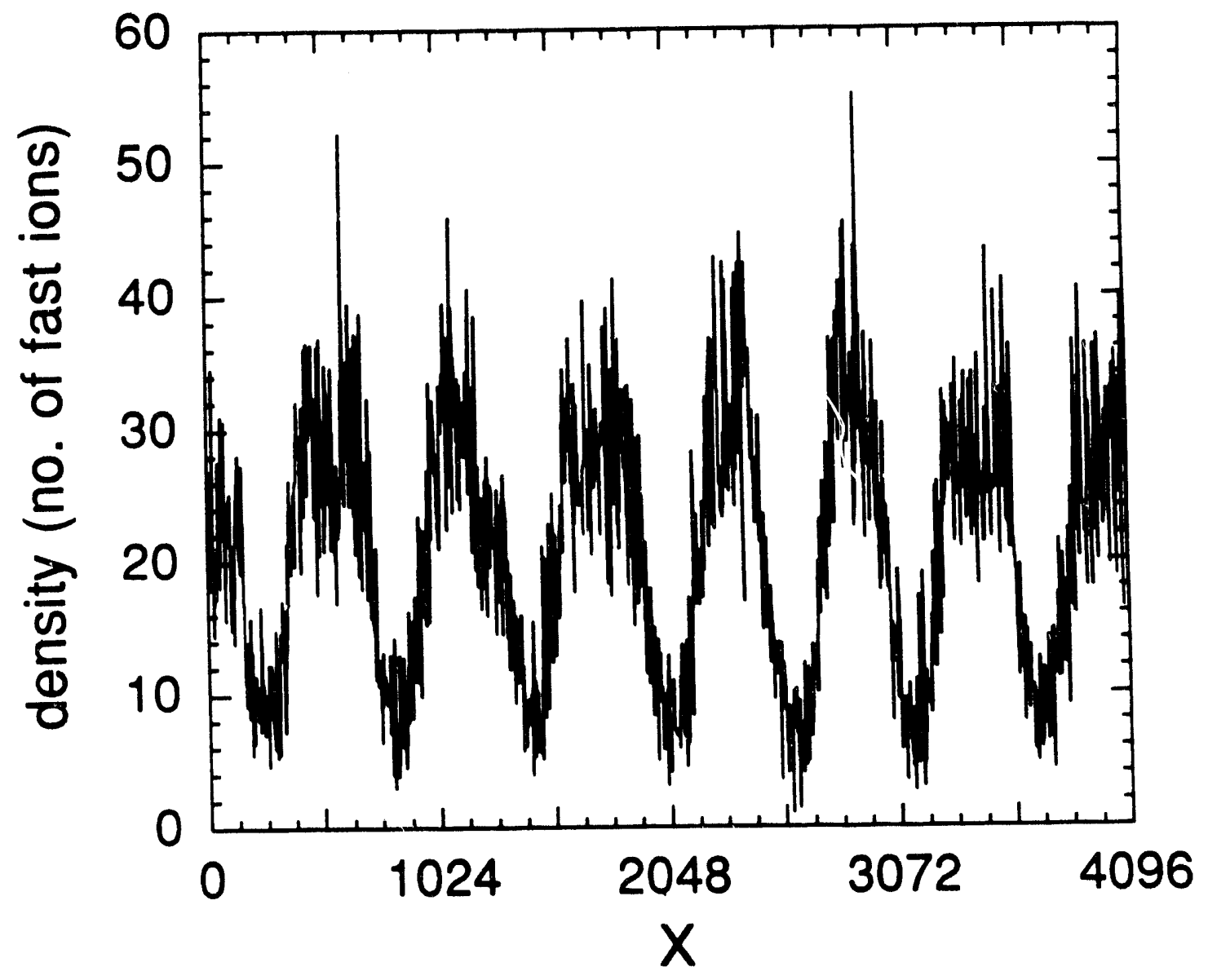

Fig. 4. by Chen 

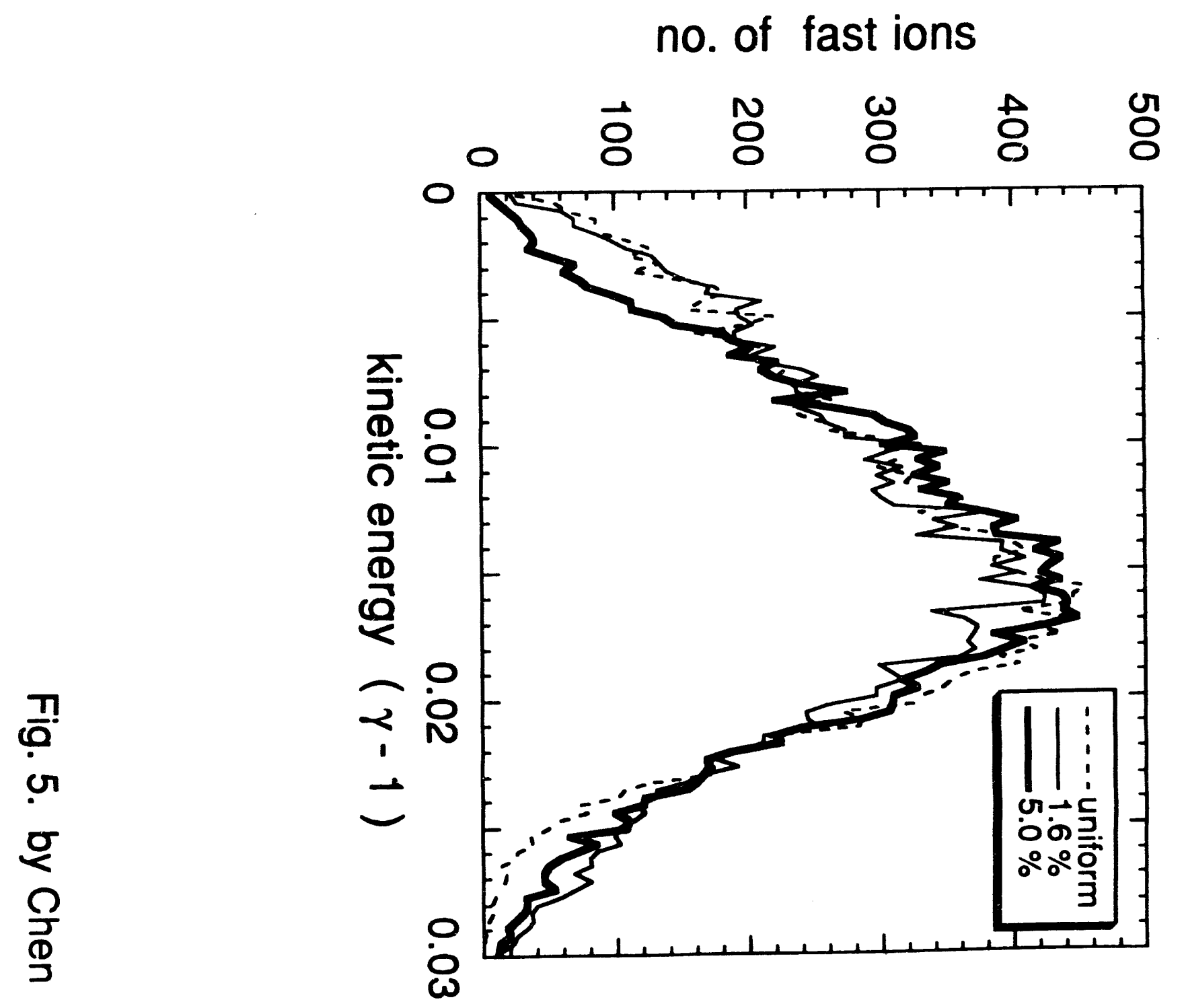


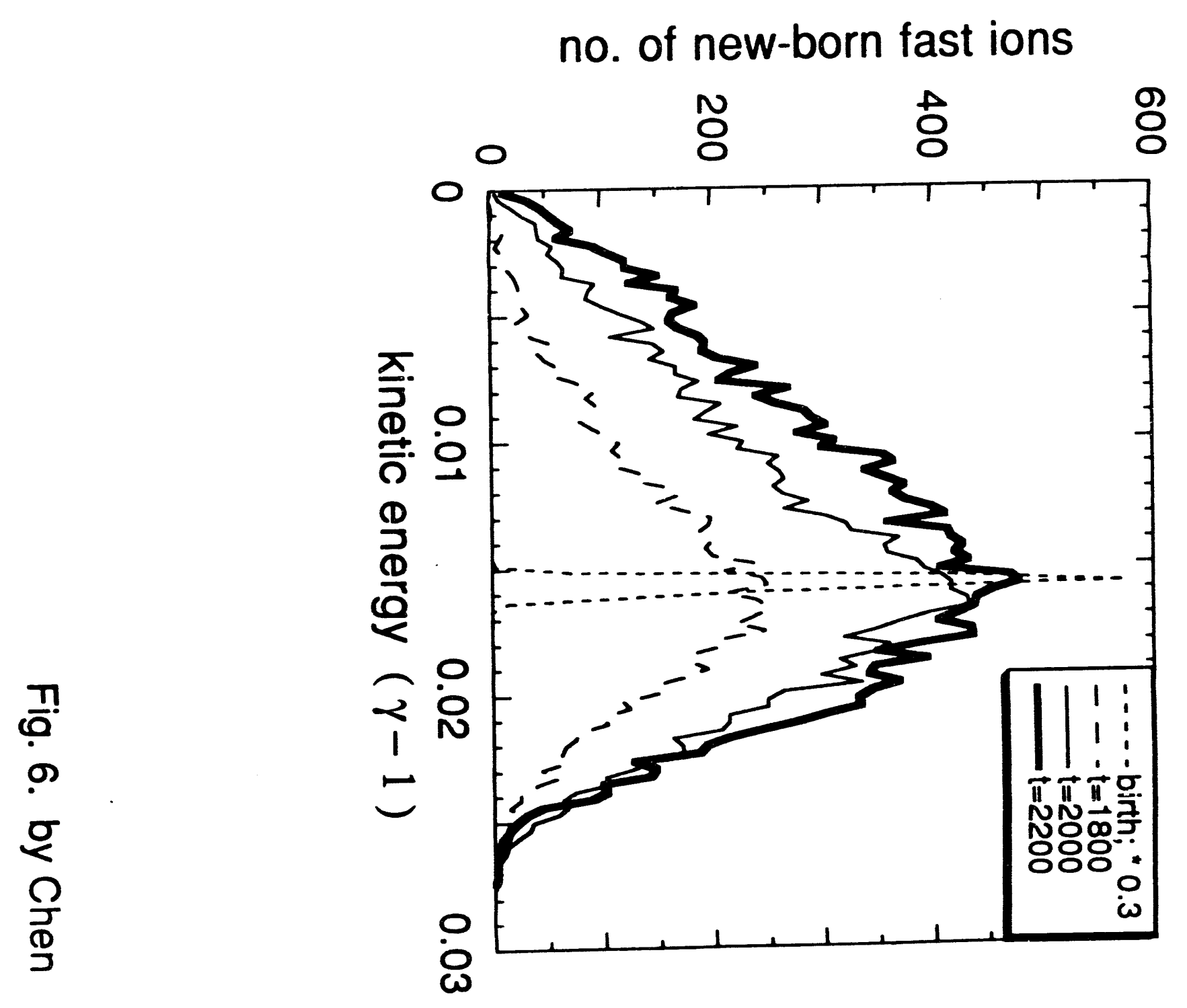



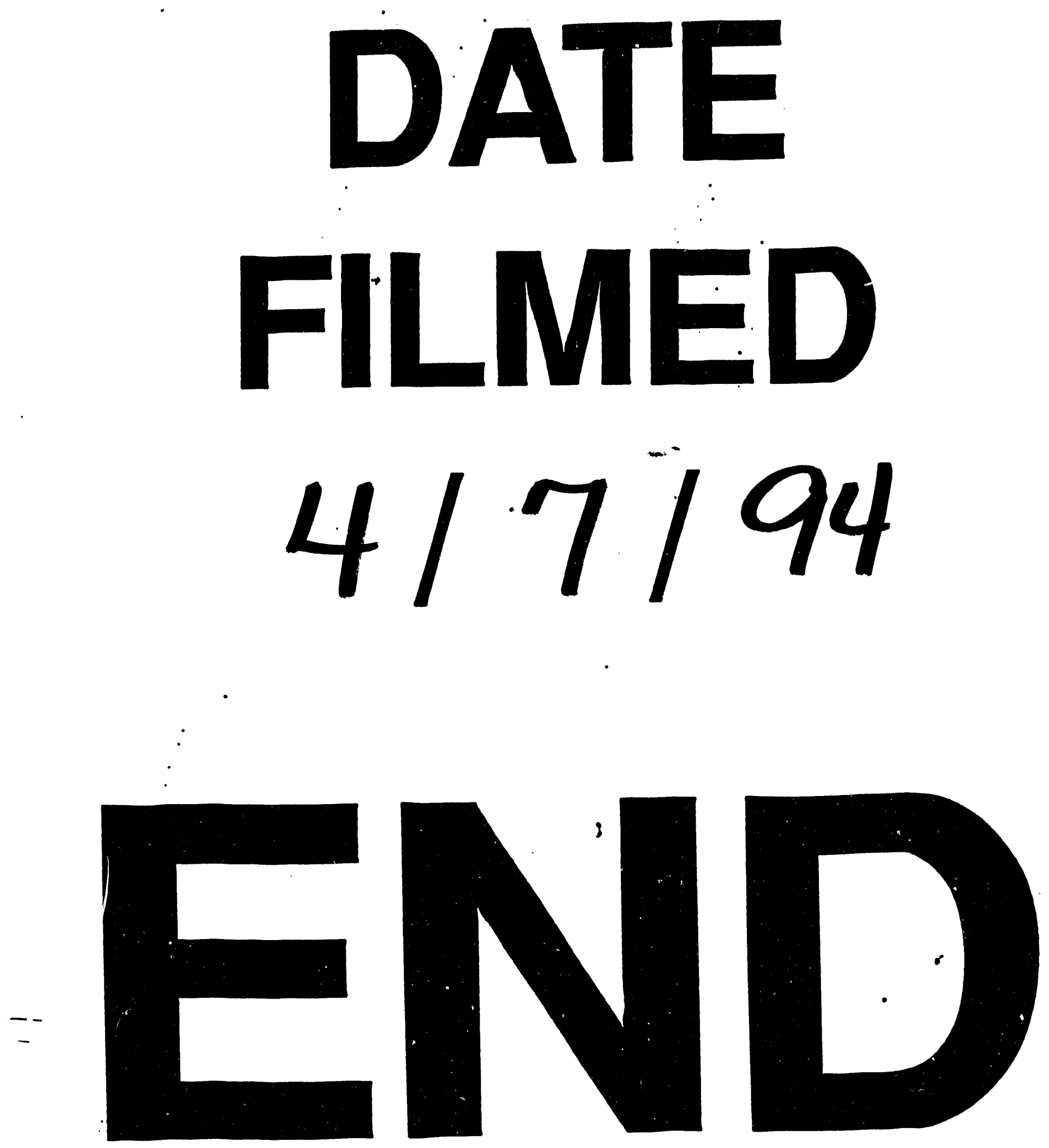
